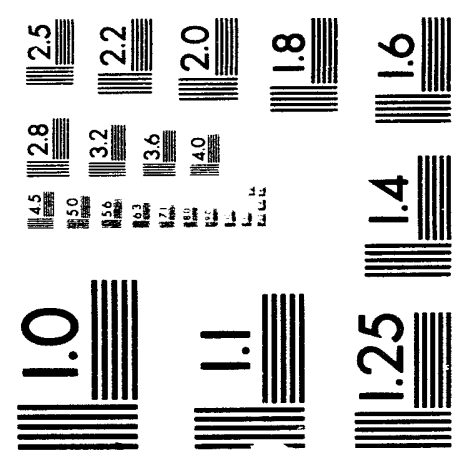



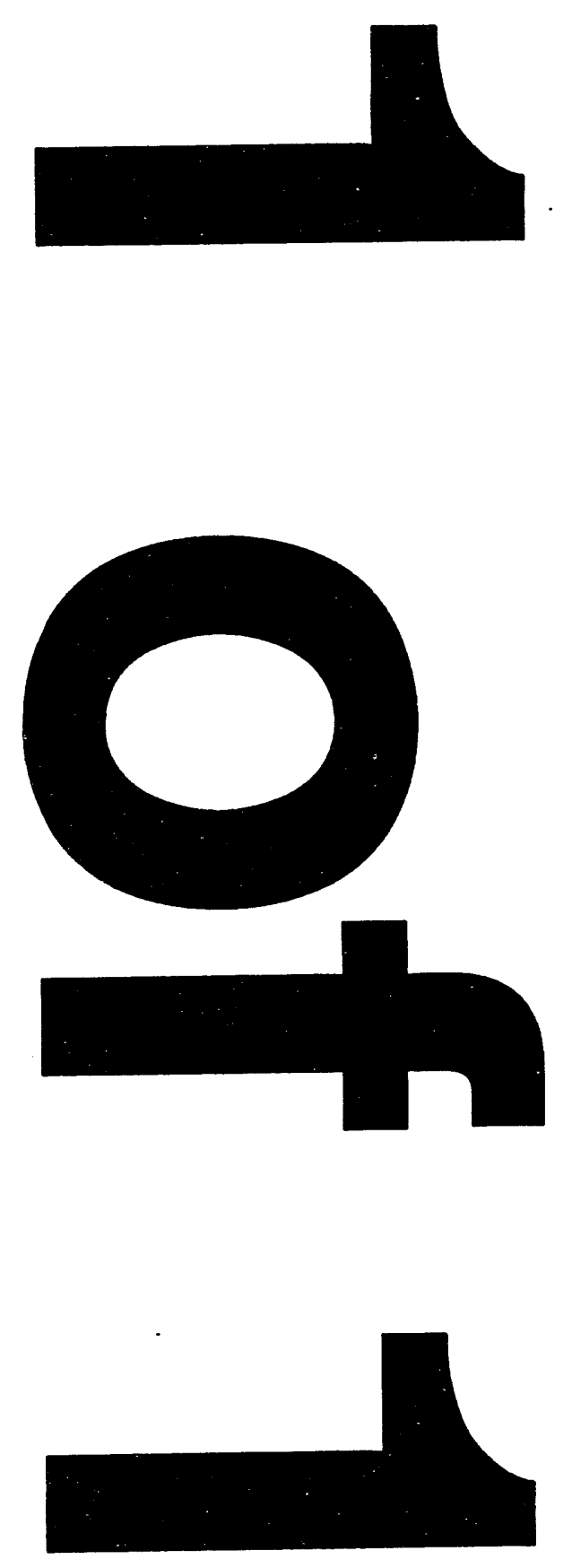


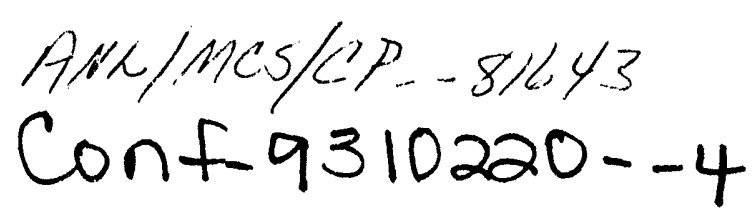

\title{
The PRISM Project: Infrastructure and Algorithms for Parallel Eigensolvers*
}

\author{
Christian Bischof ${ }^{\dagger}$ Steven Huss-Lederman ${ }^{\ddagger}$ Xiaobai Sun ${ }^{\dagger}$ Anna Tsao ${ }^{\ddagger}$ \\ †Argonne National Laboratory, Argonne, IL 60439. \\ ‡Supercomputing Research Center, Bowie, MD 20715.
}

\begin{abstract}
The goal of the PRISM project is the development of infrastructure and algorithms for the parallel solution of eigenvalue problems. We are currently investigating a complete eigensolver based on the Invariant Subspace Decomposition Algorithm for dense symmetric matrices (SYISDA). After briefiy reviewing the SYISDA approach, we discuss the algorithmic highlights of a distributed-memory implementation of an eigensolver based on this approach. These include a fast matrixmatrix multiplication algorithm, a new approach to parallel band reduction and tridiagonalization, and a harness for coordinating the divide-and-conquer parallelisin in the problem. We also present performance results of these kernels as well as the overall SYISDA implementation on the Intel Touchstone Delta prototype and the IBM SP/1.
\end{abstract}

\section{Introduction}

(omputation of eigenvalues and eigenvectors is an essential kernel in many applications, and several promising parallel algorithms have been investigated $[29,24,3,27,21]$. The work presented in this paper is part of the PRISM (Parallel Research on Invariant Subspace. Methods) Project, which involves researchers from Argonne National Laboratory, the Supercomputing Research ('enter, the University of California at Berkeley, and the Iniversity of Kentucky. The goal of the PRISII project is the development of algorithms and software for solving large-scale eigenvalue problems based on the invariant subspace de-

- This paper is PRISM Working Note \#12, available via anonymous ftp to $f$ tp.super org in the direstory pub/prism.

This work was partially supported by the Applied and Computational Mathematics Program, Advanced Research Projects Agency, under Cintract D.M28E04120, and by the Office of Scientific Computing, I'.S. Department of Energy, under Contract W-31-109-Eng-3x. Access to the Litel Touchistone Delta System operated by ('altech on behalf of the Concurrent Supercomputing ('onsortium was provided by .V.SF.

Typeset by A,S - TEX composition approach originally suggested by Auslander and Tsao [1].

The symmetric invariant subspace decomposition algorithm (SYSDA) for an $n \times n$ symmetric matrix $A$ proceeds as follows.

Scaling: Compute upper and lower bounds on the spectrum $\lambda(A)$ of $A$ and compute $\alpha$ and $\beta$ such that for $B=\alpha A+\beta I$ we have $\lambda(B) \subseteq[0,1]$, with the mean eigenvalue of $A$ being mapped to $\frac{1}{2}$.

Eigenvalue Smoothing: Let $p_{i}(x), i=1,2, \ldots$ be polynomials such that $\lim _{i \rightarrow \infty} p_{i}([0,1])=\{0,1\}$, that is, in the limit all values are mapped to either 0 or 1. Iterate

$$
C_{0}=B, C_{i+1}=p_{i}\left(C_{i}\right), i=0,1, \ldots,
$$

until $\left\|C_{i+1}-C_{i}\right\|$ is numerically negligible (in iteration $k$, say).

Invariant Subspace Computation: Find an orthogonal matrix $[U, V]$ such that the columns of $U$ and $V$ form orthonormal bases for the range space of $C_{k}$ and its complementary orthogonal subspace, respectively. That is, $U^{T} U=I, V^{T} V=I, U^{T} V=0$, and the range of $C_{k} U$ is $U$.

Decoupling: Update the original $A$ with $[l, V]$, i.e.. form

$$
\left[U^{\prime}, V\right]^{T} A\left[l^{\circ}, V\right]=\left(\begin{array}{ll}
A_{1} & \\
& A_{2}
\end{array}\right) \text {. }
$$

Since the invariant subsfaces of any matrix polynomial of a symmetric matrix $A$ are also invariant subspaces of $A$, the columns of $U$ and $V$ span complementary invariant subspaces of $A$, and hence their application to $A$ decouples the spectrum of $A$. The subproblems $A_{1}$ and $A_{2}$ can now be solved independently and the algorithm applied further recursively. with the number of subproblems doubling at every step. If eigenvectors are desired as well, we also update the current eigenvertor matrix. Orthogonality of 


\section{DISCLAIMER}

This report was prepared as an account of work sponsored by an agency of the United States Government. Neither the United States Government nor any agency thereof, nor any of their employees, makes any warranty, express or implied, or assumes any legal liability or responsibility for the accuracy, completeness, or usefulness of any information, apparatus, product, or process disclosed, or represents that its use would not infringe privately owned rights. Reference herein to any specific commercial product, process, or service by trade name, trademark, manufacturer, or otherwise does not necessarily constitute or imply its endorsement, recommendation, or favoring by the United States Government or any agency thereof. The views and opinions of authors expressed herein do not necessarily state or reflect those of the United States Government or any agency thereof. 
the eigenvectors is guaranteed due to the pxclusive use of orthogonal transformations.

Note that we have considerable freedom in implementing SYISDA, in particular with respect to choosing the polynomials $p_{i}$ as well as the method for computing the invariant subspaces. We also mention that any other method that produces invariant subspaces, such as approximation methods for the matrix sign function $[12,13,20,2]$, could be used in the Eigenvalue Smoothing step as well. As in [26], we use predominantly the first incomplete beta function $3 x^{2}-2 x^{3}$ in our implementation. The experiments in [26] also confirm the numerical robustness of SYISDA.

While the SYISDA algorithm can be used to compute a full eigendecomposition, it is worthwhile to point out certain mathematical features that distinguish it from other approaches:

Ordering of Eigenvalues: Assuming that $p$ maps all eigenvalues in $[0, a)$ to 0 and all eigenvalues in $[a, 1]$ to $1, \lambda\left(A_{1}\right)$ contains all the eigenvalues of $\mathbf{A}$ that are smaller than $\frac{a-y}{a}$, and $\lambda\left(A_{2}\right)$ contains the rest. Hence, if one is only interested in eigenvalues in a certain part of the spectrum, one need not further resolve diagonal blocks corresponding to uninteresting parts of the spectrum.

Subspaces before Eigenvalues: SYISDA is primarily an algorithm that computes and refines invariant subspaces. If such a subspace has become one-dimensional, an eigenvalue/eigenvector pair has been found. However, if one is only interested in finding an orthogonal basis for the subspace spanned by a certain set of eigenvectors, there is no need to expend the effort to compute all eigenvalues. and one can terminate the divide-and-conquer procedure when the subspace corresponding to the desired eigenvalue range has been identified.

No Problems with Repeated Eigenvalues: Clusters of rigenvalues are quickly gathered in the same subproblem and are. in fact, likely to increase the sperel of convergences of the Eigenvalue Smoothing step. The orthogonality of eigenvectors is not atfected at all by repeated eigenvalues

The two key primitives of the algorithm are matrixmatrix multiplication. which accomts for the majority of the computation and computation of the range and mull space of a matrix having eigenvalues cluslered around zero and one. The sequential complexity of SYlSIA, when applied to dense matrices, is considerably greater than that of other algorithms. Nonetheless, the algorithu is promising from both a scalability and a mumerical point of view. First. since most of the computation is in matrix multiplication, high efficiencies and near optimal speedups can be expected on large problems. Second, since the algorithm performs only orthogonal transformations, orthogonality in the computed eigenvectors is guaranteed.

The paper is organized as follows. The next section briefly discusses the implementation of matrix-matrix multiplication. Section 3 discusses the rank-revealing tridiagonalization algorithm employed for the Invariant Subspace Computation step. It is based on the successive band reduction (SBR) framework developed by Bischof and Sun [9] and, while completely general, derives significant benefit from the special eigenvalue structure of the matrices at hand [8]. Section 4 discusses the overall divide-and-conquer strategy employed to orchestrate the various subproblems and presents preliminary performance results of the first SYISDA implementation on the Intel Touchstone Delta. We conclude with our findings, in particular with respect to the impact of data layout on the design of scalable libraries for the support of matrix compuiations, and describe how the kernels we have developed form the infrastructure for a "library" of parallel eigensolvers.

\section{Matrix Multiplication}

As noted previously, the computational cost for SYISDA is dominated by dense matrix multiplication. Hence, its performance depends heavily on having a scalable matrix multiplication code for our initial target machine, the Intel Touchstone Delta. We have developed a distributed matrix multiplication code that calculates the products $C^{\circ}=\operatorname{cx} A B+36^{\circ}$ and $C^{\circ}=\operatorname{rr}^{t} B+3 C^{\circ}$ in double precision. Our objectives were to

(1) provide a highly efficient algorithm suitable for use by SYISDA,

(2) strive for high performance on large square matrices.

(3) provide robust performance for mesh contigurations with poor aspect ratios, and

(4) use an algorithm whose kernels match well with the expected capabilities of future marhines.

As usual, a critical issue in achieving high efficiency is data locality, i.e. maximmm reuse of data in Hoating point computations. To this end. we utilize the highly optimized assembly-coded double precision general matrix multiplication. I) ( EMMM. on single nodes. It is capable of sustaining 36.5 . Mflops (empirically determined using Release 1.4 of Intel $\mathrm{XX} / . \mathrm{I}$ ()S and related software) of the 40 Mflops possible. ()n the 
Delta, optimal single node performance for DCEMM often decreases significantly as the matrix shapes become less square or when matrix granularities become finer. Thus, our first general rule is to strive for matrices having all their dimensions as large as possible in local computations.

Let us first consider the case of square meshes. In the parallel SBR strategy, it is extremely desirable from both a performance and ease of programming point of view to have row and column blocks physically spread across processor columns in a torus wrap fashion (see [6] for example). Furthermore, the divideand-conquer strategy we use assumes that the blocks of the matrix are spread out fairly evenly across the mesh in such a way that the generated subproblems remain spread out all over the mesh. Therefore, twodimensional torus wrap is ideal. For the purposes of matrix multiplication alone, optimal performance occurs when each node has the same amount of data for each matrix; the use of torus wrapping is neither an advantage nor a disadvantage. The situation changes, however, when we try to generalize two-dimensional torus wrap to nonsquare meshes. We found that the choice of data layout has a significant effect on both the ease of programming and granularity of local computations. We chose to use a generalization of twodimensional torus wrap on non-square meshes, known as virtual two-dimensional torus wrap, that resulted in simpler, more easily tuned algorithms. Some of our preliminary findings are discussed in [6] and [22].

The Broadcast-Multiply-Roll (BMR) algorithm [16, 11. 28] has been demonstrated to scale extremely well un loosely coupled square processor meshes and ust's two readily portable communication kernels: onelimensional broadcast and roll. We have implemented a variant of BMR in (' using communication primitives highly suited to the Delta $[22]$. In particular, since the Delta does not. effectively overlap communication and computation, the algorithm we chose is highly synchronous.

() Ir B.MR variant is able to deal with arbitrary rectangular meshes and matrix dimensions. ()ur code can deal with a variety of tifferent virtual two-dimensional torus blocking schemes by means of a user-passed function providing block size information. The implementation gllarantess that all matrix operands end up in place. Details of our implementation are discussed in $[22]$.

In Figure 1. we give the total performance for our distributed matrix multiplication on square submeshes of the Delta for matrices having matrix size $100 \times 100$, $.100 \times 900$, and $550 \times 500$ on each procesisor. In particufar, war corle has achieved a parallel efficiency of $86 \%$.

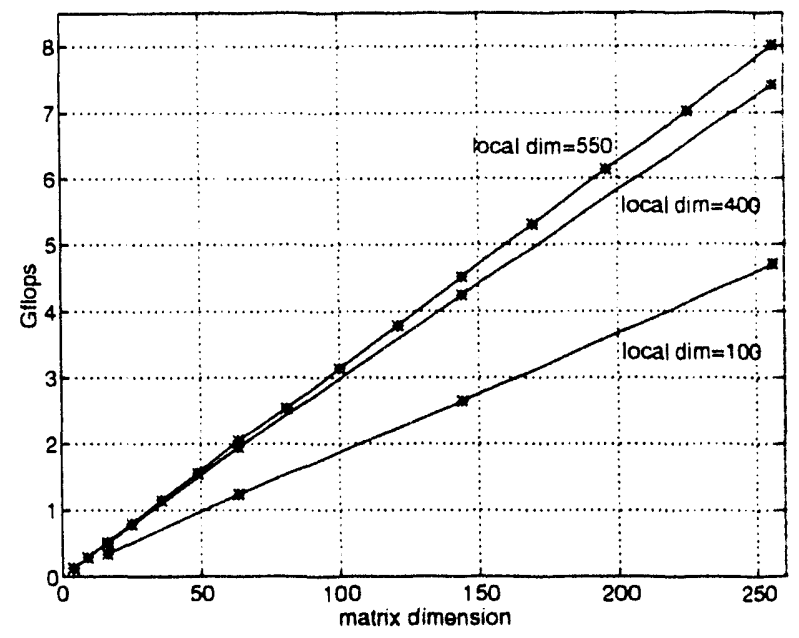

Figure 1. Total performance of matrix multiplication on Delta

with overall peak performance in excess of 8 (iflops on 256 nodes for an $8800 \times 8800$ double precision matrix and has demonstrated robust performance for nonoptimal mesh aspect ratios.

While our orientation was machine-specific and aimed at optimization rather than portability, our BMR variant will port well to other distributed architectures such as the IBM SP/I.

\section{Successive Band Reduction}

To find the orthogonal transformation that decouples $A$, we have to find the range and mull space of a matrix that has only two distinct eigenvalues, 0 and 1 . For ease of reference, we henceforth call matrices with this special property PRISM matrices. As it turns out. the Invariant Subspace Computation step can be achieved essentially via a tridiagonalization of $\left({ }^{\prime} k\right.$. The key observation is that, under some very general conditions, a band matrix having only two distinct eigenvalues and bandwidth $n / 2^{j}$ must be block diagonal. with each block being of size at most $n / 2^{j-1}$. In particular, a tridiagonal matrix with such a spectrum is block diagonal with blocks of size at most $2 \times 2$. Hrnce. after the matrix has been reduced to tridiagonal form. one only needs to solve some (completely independent $2 \times 2$ eigenvalue problenis to obtain the desired invariant subspaces. These issues, as well as some of thr subtle numerical issues arising in this context, are discussed in [8].

It is important to realize that, mulike ofler approaches for computing so-called rank-revealing fac- 
torizations $[4,5,10,30]$, tridiagonalization does not involve any data-dependent pivoting strategies. In particular, in the parallel setting, the predictability of data flow greatly contributes to simplicity of implementation as well as to the ability to overlap communication and computation.

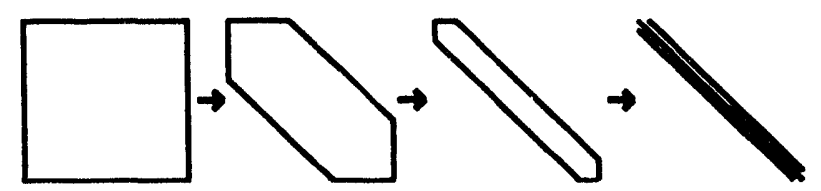

Figure 2. Reduction to tridiagonal form by a sequence of band reductions

In order to reduce the given matrix to tridiagonal form, we employ a variant of the successive band reduction framework suggested by Bischof and Sun [9], which eliminates subdiagonals of $C_{k}$ in a piecemeal fashion as illustrated in Figure 2. In comparison, conventional Householder tridiagonalization approaches [17] or block variants thereof [14] eliminate all subdiagonals at one time. This traditional approach also underlies the parallel implementations described in [19] and [1.5]. The SBR variant used in our implementation is discussed in detail in [7].

In our particular version of SBR, we first reduce $C_{k}$ to a banded matrix of bandwidth $n b$, using block orthogonal transformations with blocksize $n b$ and then, in a second step, reduce the band matrix of bandwidth $n b$ to tridiagonal form. The first step maximally exploits block transformations, and the hope is that the little work that is left to be done in the second step (from a Hop count point of view) does not add much to the overall complexity. In the context of SYISDA, we also expect to be able to skip large numbers of the orthogonal transformations, since the block diagonality of the matrices we will generate should result in many transformations that would act on columns that are already negligible and hence need not be performed.

(One issue that is critical is the need to repackage the banded matrix remaining after the first initial reduction step into a more compact form that allows us to atcess adjacent entries of the band efficiently. For example. in a serial implementation, entries $(i+n b, i)$ and $(i+1+n b, i+1)$ are at least $n-1$ storage locations apart if an $n \times n$ matrix has been stored as the usual t.wo-dimensional array. Hence, the final band reduction scheme would exhibit no data locality and would, as a resilt. suffer severe performance penalties 6n rache-based architertures. Even worse performance prolalties would result in the parallel setting, as very little data are left for every process to work on. but a lot of communication is needed to access successive matrix elements and perform bulge chasing. To implement the second band reduction step, we redistribute the remaining band into packed storage and then employ a variant of an algorithm suggested by Lang [25] to reduce the band matrix to tridiagonal form. We made several modifications to Lang's algorithm to improve the memory locality of the algorithm, and details will be reported in a forthcoming paper.

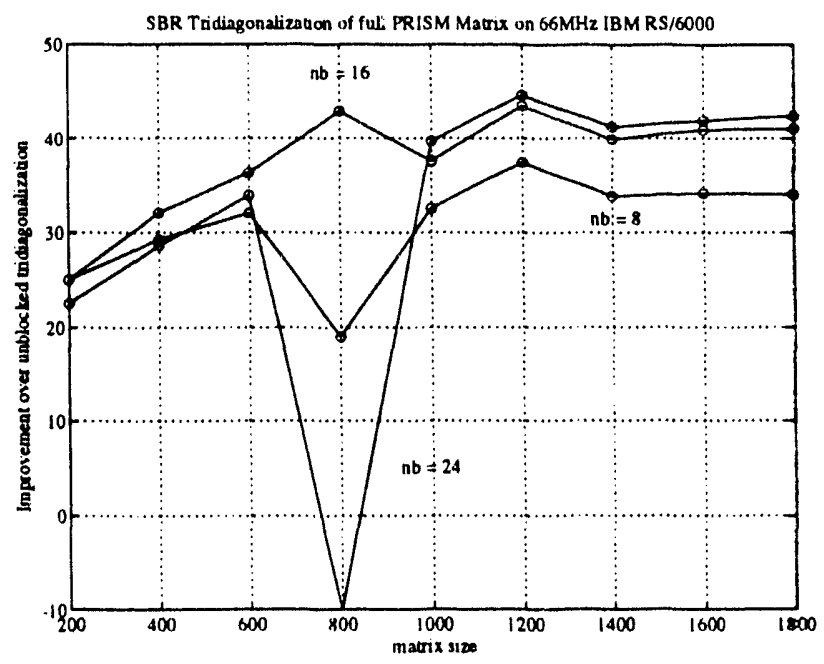

Figure 3. Improvement over standard tridiagonalization approach through SBR for PRISM matrices on IBM R.S $/ 6000$

Our SBR approach significantly outperforms the usual tridiagonalization approach on SYISDA matrices. For example, as is shown in Figure 3, running on a 66-Mhz IBM RS/6000 with 128 . MB Bytes of memory and using assembler-coded BLAS, our SBR approach runs a good $30 \%$ faster than the standard tridiagonalization procedure. Note that these times reflect both the time for the reduction from full to tridiagonal form and the accurnulation of the orthgonal transformation matrices. Ignoring the usual timing variations one is bound to expect on a non-dedicated system, the blorksize chosen does not seem to have a significaut. effert on the overall performance.

As hinted earlier, we profit from the underlyins structure of the matrices arising in SYISDA. Figur 4 shows how many reductions we can skip in the first and second band reduction steps, where we first rliminate $d$ subdiagonals $(d \geq n b=16)$ of a $400 \times 400$, dense PRISM matrix and then reduce the resulting matrox 

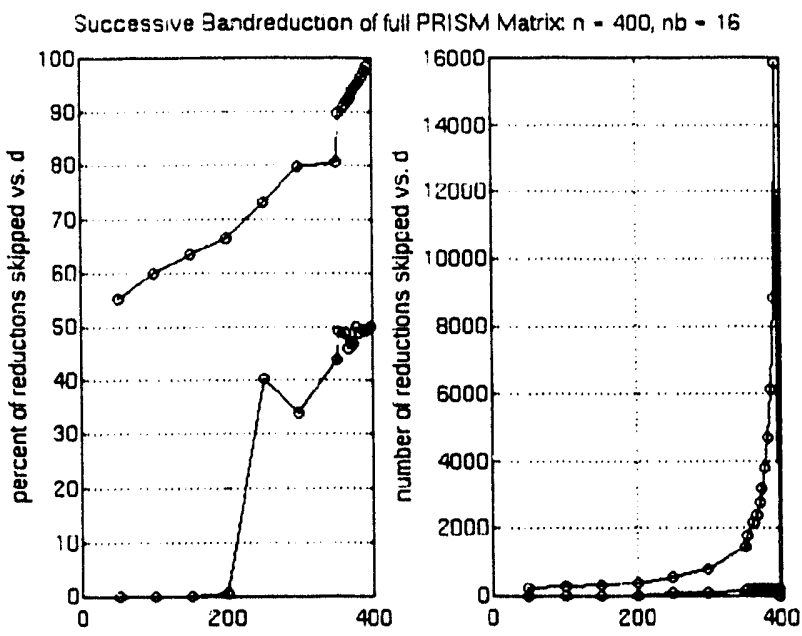

top: 2nd band reduction bottom: 1 st band reduction

FIGURE 4. Work savings due to exploitation of eigenvalue structure via SBR

to tridiagonal form. While a standard tridiagonalization approach, which eliminates all subdiagonal entries in one shot, would never incur any numerically zero columns and hence never skip a transformation, SBR can take advantage of the special block structure exhibited by banded matrices with only two eigenvalues. The left plot shows the percentage of transformations we could skip for a particular value of $d$, the right plot shows the total number of transformations skipped. We see that it is beneficial to reduce the matrix to a relatively narrow band in the first step. One benefit is that we get closer to the desired tridiagonal form, and another is is that we skip just about half the transformations in the first band reduction step, and over $90 \%$ in the second band reduction step. On the other hand, reducing a matrix to too narrow a band is counterproductive, as the blocksize becomes too small and the number of transformations needed for bulge chasing grows considerably. Based on these experiments, we consider an initial reduction to between $x$ and 24 bands a reasonable choice.

The parallel implementation of this SBR scheme is in progress, with the first step having been completed. In fact. we implemented a general band reduction tool that allows us to reduce a mat rix of bandwidth $n b * k$ to a unatrix of size $n b * l$, with $l \leq l \leq k-1$. Furthermore, $n b$ is the block size used for the blocked torus wrap mapping and also the block size used for orthogonal transformations. Blocked reductions of full matrices to. narrow bands and the unblocked tridiagonalization of a full matrix are special instances of this code. $T c$ develop is portable code and to allow a maintiainable implementation, we chose to base our implementation on the Chameleon parallel programming tools [18].

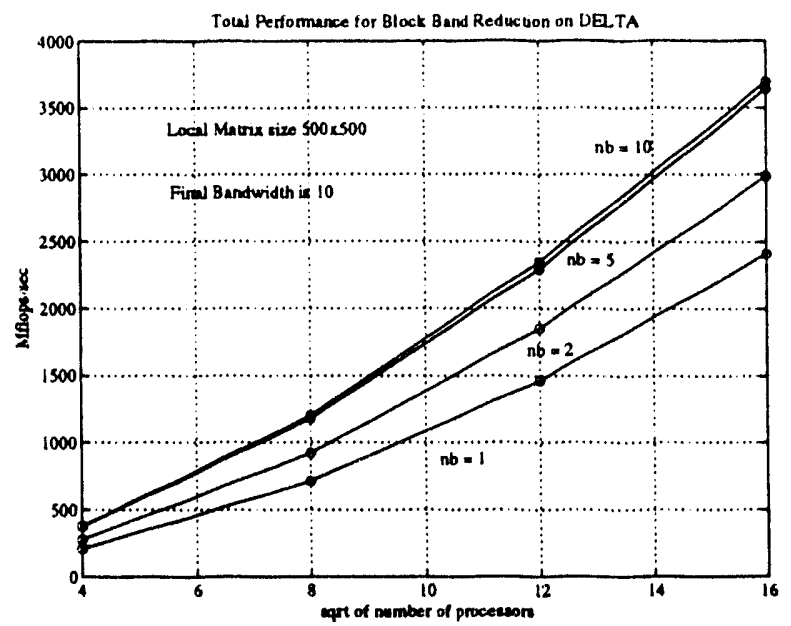

Figure 5. Total performance of blocked band reduction code on Delta

The performance of this code is rather promising, and its performance on the reduction of a full random matrix to bandwidth 10 and the accumulation of orthogonal transformations on the Intel Touchstone Delta are shown in Figure 5 . In these experiments. the matrix size on each processor was kept constant at $500 \times 500$, and the execution rates are based on the standard symmetric flop count of $\left(8 n^{3}\right) / 3$. These experiments were performed in double precision. We recall that for random matrices, no transformations can be skipped.

\begin{tabular}{|r|r|r|r|r|}
\hline$n$ & $n b$ & $b$ & Mflops/proc & Total Mflops \\
\hline 1000 & 1 & 20 & 10.1 & 162 \\
\hline 1020 & 15 & 15 & 18.7 & 299 \\
\hline 2000 & 1 & 20 & 10.0 & 160 \\
\hline 2000 & 10 & 10 & 26.2 & 419 \\
\hline 3000 & 1 & 10 & 10.9 & 174 \\
\hline 3000 & 15 & 15 & 33.7 & 53.59 \\
\hline 4000 & 20 & 20 & 38.2 & 611 \\
\hline
\end{tabular}

TABLE 1. Preliminary performance results on 16 -node $\mid \mathrm{B} . \mathrm{S} \mathrm{SP} / 1$

Some rather preliminary performance results on ars IBM SP/l, using the EVI-H transport layer, are shown in Table 1 . Here $n$ is the overall matrix size, $n b$ is the block size used, $b$ is the number of bands the full 
matrix is reduced to, and the last two columns are perprocessor performance and overall performance for a double-precision run. again based on the standard flop count. These results should be taken as a lower bound on the ultimate performance, as the performance of the IBM software and of (hameleon are improving rapidly.

We see that, even now, the performance of the blocked code is superior to that of the unblocked code on both Delta and SP/1. In particular, on the SP/1 the blocked code outperforms the unblocked one by a wide margin. For example, in the time that we can reduce a $2000 \times 2000$ matrix to tridiagonal form using the unblocked approach, we can reduce a $3000 \times 3000$ matrix to bandwidth 10 , even though the latter reduction involves roughly 3.5 times as many floating-point operations.

We are in the process of implementing a parallel version of Lang's algorithm, where the banded matrix (but not the orthogonal transformation matrix) is replicated in each row of the processor mesh. (iiven the performance gap between blocked and unblocked band reduction and the savings we are likely to incur as a result of orthogonal transformations being skipped, the final parallel SBR code should be even better in comparison to the unblocked code than the serial one.

\section{Load Balancing Strategies and Per- formance Results}

The orchestration of multiple subproblems currently consists of two separate stages. The first stage encompinsies the early divides, where large subproblems are solved sequentially and the scalability of the dense matrix multiplication and rank-revealing tridiagonalization leal to high efficiencies. As pointed out in Section 1. Clusters tend to be gathered into the same subproblem. Since rarly cluster detection can greatly reduce the amount of work done, we use a simple heuristic schene that chooses whichever of $A_{1}$ or $A_{2}$ has all of its eigenvalues on the same side of 1$)$ as the mean eigenvalue of $A$. A running estimate. A. of the largest motan rigrenvalur in magnitude from alreacly completed divides is kept. When the bounds used in the Scaling step of SYISDA indicate that all the eigenval-

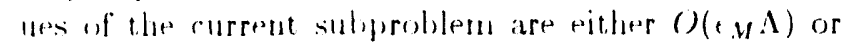
within ()$(,, A)$ of each other $(e, y$ is the machine epsilon). then the subpreblem is declared to have clustered rigenvalues and to he "rlente."

Through the use of ewo-dimensional horus wrap, no data redistribution is required between divides. As the suhproblem size decreases, the proportion of the total time refpuired for commmonication during individual matrix multiplications increases and the granularity of local computation decreases. However, this approach guarantees near-perfect load balancing in the costly early stages. A discussion of the rationale used to arrive at this scherne is presented in [2:3].

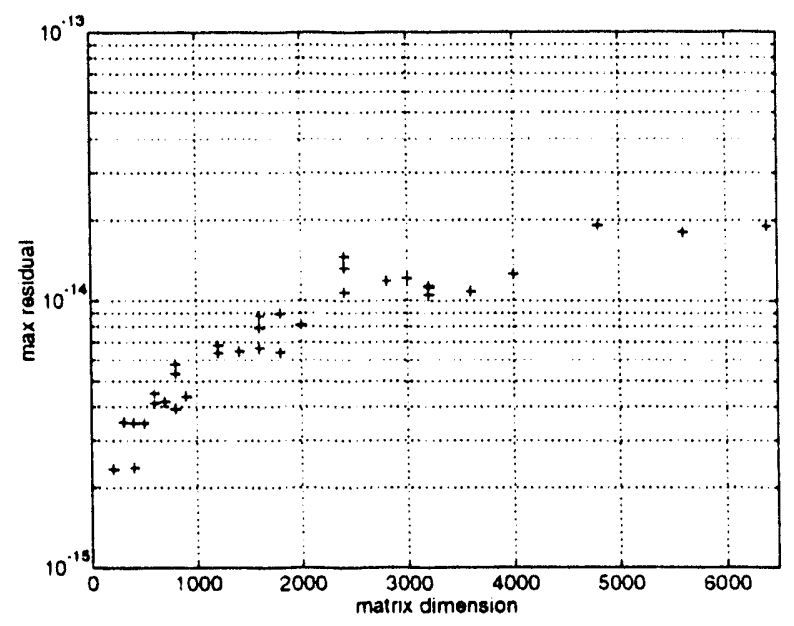

(a) Residuals

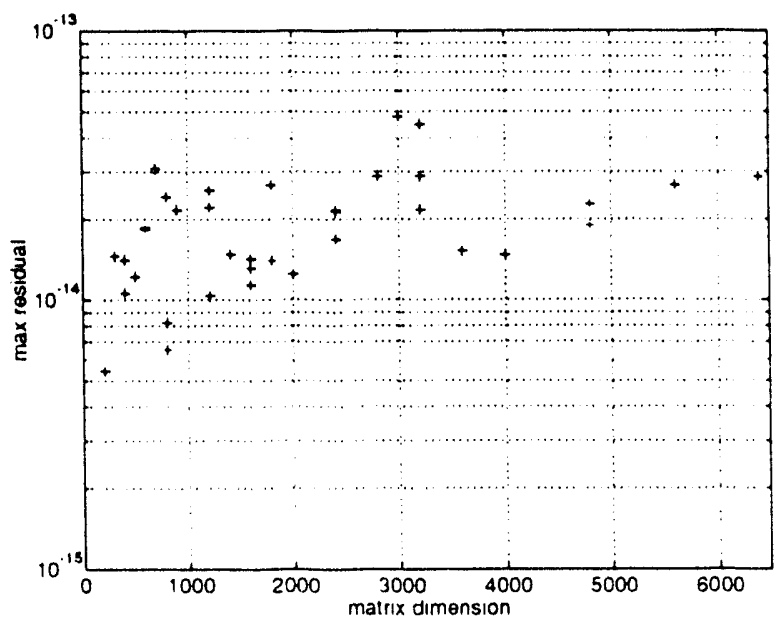

(b) Eigenvector orthogonality

FICiURE 6. Numerical accuracy of SYISDA

As the subproblem size decreases. there is a point probably machine-dependent, at which communications overhead makes it impractical to solve that sul,problem over the entire mesh. The amomnt of work required to find the eigensolution of the remaining sul.problem is very small, hut the cost of the update of thr. eigenvector matrix $Z$, whose leading dimension is still the size of the original matrix $A$, is still substantial. The second stage, or end game, handles the small sul. problems remaining, exploiting parallelism at two les. 
els: first, by solving individual subproblems in parallel on single nodes and second, by performing distributed updates of the accumulated eigenvector matrix. The strategy used in the end game is also described in [23].

Numerical testing in double precision of our parallel algorithm, using the unblocked version of the SBR code, is currently in progress. Easily generated symmetric test cases with uniformly distributed eigenvalues of dimensions 100-6400 have been tested so far. Accuracy in the residuals for a given matrix $A$ is measured by computing the maximum normalized $\infty$-norm residual

$$
\max _{i} \frac{\left\|A Z_{i}-\lambda_{i} Z_{i}\right\|_{\infty}}{\|A\|_{1}}
$$

where $Z_{i}$ is the computed eigenvector corresponding to the eigenvalue $\lambda_{i}$. We also measured the departure from orthogonality residual given by

$$
\max _{i, j}\left|\left[Z^{t} Z-I_{n}\right]_{i j}\right|
$$

to verify that the computed eigenvectors were, indeed, orthonormal. Here $Z$ is the matrix of eigenvectors. Figure 6 shows that in terms of both accuracy in the residuals and orthogonality of the eigenvectors, SYISDA is performing well. We have done some limited testing on Wilkinson matrices and have thus far encountered no problems with accuracy.

\begin{tabular}{|r|r|r|r|r|}
\cline { 2 - 5 } \multicolumn{1}{c|}{} & \multicolumn{4}{|c|}{ time (min.) } \\
\hline$n$ & $p=4$ & $p=8$ & $p=12$ & $p=16$ \\
\hline 1600 & 23.16 & 7.98 & 4.97 & 3.70 \\
\hline 3200 & - & .50 .96 & 27.97 & 18.60 \\
\hline 4800 & - & - & 53.11 & 52.60 \\
\hline 6400 & - & - & - & 115.51 \\
\hline
\end{tabular}

TABLE 2. Time for complete eigensolution of $n \times n$ matrix on $\mu \times p$ submesh of Delta

Table 2 gives the solution time in minutes for test matrices of sizes $1600,3200,4800,6400$ on $\mu \times p$ meshes where $p=4.8 .12,16$.

Figure 7 shows the total performance of SYISDA for our test matrices on square submeshes of the Delta for matrices having matrix dimensions of 100, 200 . 300, and 400 on rach processor. ('learly. SYYSDA scales extremely well. We see from Figure 1 that our distributed matrix multiplication runs at about 7.4 (iftops on a $16 \times 16$ mesh for $6400 \times 6400$ matrices: SYISDA achieves 6.4 (iflops, about $86 \%$ of what we believe to be the maximum prosible performance for this algorithm.

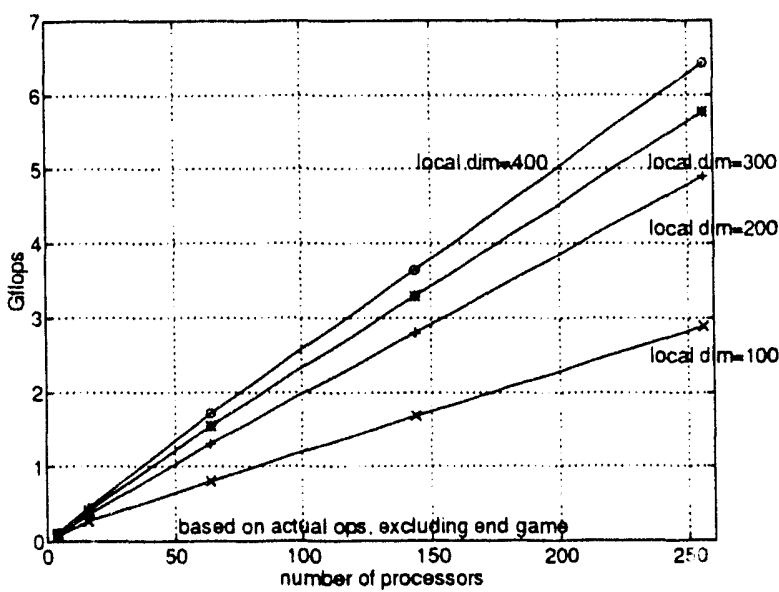

Figure 7 . Total performance of SYISDA on square submeshes of Delta

Our assembled SYISDA code currently runs only on square submeshes of the Delta using the unblocked SBR code. We expect its performance to improve when the blocked SBR has been completed. We are in the process of porting the code to other architectures and plan to generalize the code to non-square processor meshes in the future.

\section{Data Layout and Library Considera- tions}

Our work has reinforced the need for systemic support of basic communication kernels that are inevitably required when data are physically distributed. Because communication is highly machine-dependent, portability and compatibility with other software are often obtained at the price of performance or increased software developnent effort. Support for data redistribution is needed at two levels. The first is to support basic operations that are needed within the same data layout. In our rase, the use of data layouts with symmetric placement of rows and columns led to simpler algorithms than with other layouts. without sacrific. ing the performance design objectives. However since the data layout we used (as is the case for most data layouts) is not supported in a systemic manner, many "low-level" communication routines had to be written to perform what should be compiler-level operations. For instance, routines were needed for the basic operations described below.

Srattering: The problem to be solved must be distributed on the processors in the layout used.

Redistribution: In both the SBR and the end game 
blocks of data scattered over one submesh had to be redistributed or replicated over a different submesh, sometimes in a different layout.

Layout conversion: In both $\mathrm{SBR}$ and for the invariant subspace update, data redistribution is required whenever the matrix being updated is not aligned with the update matrix.

Specialized routines had to be developed to deal with each of these situations. The second level of support should be in accommodating different layouts. Even within the torus wrap data layout, changing the block size requires significant data movement. Without such support, more specialized routines would be required. In fact, all the types of data movement described here pervade numerical computation and should be supported in future parallel languages such as High Performance Fortran. Otherwise, every user will be required to write his own specialized embedded routines. Continued lack of compiler support for basic communication kernels associated with data layout and movement will not only greatly impede library development, but will also impede the adoption of these libraries by users because of interface problems.

In our work on SYISDA, we have developed three kernels that provide the infrastructure for a "library" for parallel eigensolvers and, to a more limited extent, for numerical linear algebra computations. The first is matrix multiplication, the primitive of choice in current algorithmic design. The second, SBR, can in fact be used in conjunction with any tridiagonal or banded eigenvalue solver. Finally, the harness we have developed for managing the multiple subproblems produced by SYISDA readily transfers to any algorithm based (n) the invariant subspace decomposition approach. In particular, our approach can be readily modified to solve specialized partial spectrum problerns, including those where the eigenvalues in a specified interval are desired and those where only the invariant subspace corresponding to eigenvalues in a specified interval is soinght.

()ur pretiminary experiments indicate that our parallel inplementation of SYISDA provides excellent scaling and accuracy. In addition to continuing our studies of SYISLA and the data layout issues associated with the kernels we are interested in, we are also investigating variants of SYISDA aimed at reducing the amunnt of time spent doing dense matrix multiplication. A particularly promising algorithm, SYBISDA, uses SBR to reduce A to a llarrow band and then periodically reduces matrices in the Eigenvalue: Smoothing step to a narrow band. Multiplication of two matricess of bandwidths b only requires
$O\left(b^{2} n\right)$ work versus $O\left(n^{3}\right)$ for two dense matrices. Furthermore, the special properties of the iterates in the Eigenvalue Smoothing step result in surprisingly slow band growth in the iterated matrices. In our sequential implemeritation, using specialized routines for multiplying symmetric band matrices [31], the run times for SYBISDA are competitive with the symmetric $Q R$ algorithm. In fact, the time spent in SBR becomes the dominant time. SYBISDA uses essentially the same harness as SYISDA and the two computational kernels of SBR and banded matrix multiplication. We intend to modify our current code to obtain an implementation of SYBISDA and compare the two algorithms.

\section{Acknowledgements}

The authors would like to thank Bill Gropp and Barry Smith for their help and support with the Chameleon programming system. We also thank Bruno Lang for providing us with his serial code for a packed storage bandreduction and for some stimulating discussions on this subject. Lastly, we thank Thomas Turnbull and Elaine Jacobson for many helpful discussions during the course of this work.

\section{References}

[1] Auslander, L. \& A. Tsao, On parallelizable eigensolvers, Adv. Appl. Math. 13 (1992), 25:3-261.

[2] Bai, Z. \& J. Demmel, Design of parallel nonsymmetric eigenrouline toolbox, Fart I, Research report 92-09, University of Kentucky (Dec. 1992), (also PRISM Working Note \# 5)

[3] Berry, M. \& A. Sameh, Parallel algorithms for the singular value and dense symmetric eigenvalue problem, (SRD (1988), no. 761 .

[4] Bischof, C., A parallel $Q R$ factorization with controlled local pivoting, SIAM J. Sci. Stat. Comput. 12 (1991), 36-5i.

[5] Bischof, (. H. \&. P. (. Hansen, A blork algorithm for computing rank-revealing $Q R$ factorizations. also M('S-P251-0791, Numerical Algorithms 2 (1992), no. 3-4, 371-392.

[6] Bischof, (․ H., S. Huss-Lederman, E. M. Jacolson, X. Sun, \& A. Tsao, On the impact of HF'F data layout on the design of efficient and matntanable parallel linear algebra librartes, (private communication).

[7] Bischof, (., M. Marques, \& X. Sun, Parallel bandreduction and tridiagonalization. Proceedings, Sixth SIAM ('on ${ }^{\text {ra }}$ rence on Parallel Processing for Scientific ('omputing (Norfolk, Virginia. March 22-24, 199:3) (R. F. Sincovec, eds.), SIA.II. 
Philadelphia, 1993, (also PRISM Working Note \#8).

[8] Bischof, C. \& X. Sun, A divide-and-conquer method for tridiagonalizing symmetric matrices with repeated eigenvalues, Preprint MCS-P2860192, Argonne National Laboratory (1992), (also PRISM Working Note \#1).

[9] Bischof, C.\& X. Sun, A framework for symmetric band reduction and tridiagonalization, Preprint MCS-P298-0392, Argonne National Laboratory (1992), (also PRISM Working Note \#3).

[10] Chandrasekaran, S. \& I. Ipsen, On rank-revealing $Q R$ factorizations, Technical Report YALEU/ DCS/RR880, Yale University, Department of Computer Science, 1991.

[11] Choi, J., J. J. Dongarra, \& D. W. Walker, Level 3 BLAS for distributed memory concurrent computers, CNRS-NSF Workshop on Environments and Tools for Parallel Scientific Computing (Saint Hilaire du Touvet, France, September 7-8, 1992), Elsevier Science Publishers, 1992.

[12] Denman, E. D. \& A. N. Beavers, Jr., The matrix sign function and computations in systems, Appl. Math. Comp. 2 (1976), 63-94.

[13] Denman, E. D. \& J. Leyva-Ramos, Spectral decomposition of a matrix using the generalized sign matrix, Appl. Math. Comp. 8 (1981), 237-50.

[14] Dongarra, Jack J., Sven J. Hammarling and Dlanny (:. Sorensen, Block reduction of matrices to condensed form for eigenvalue computations, MCS-TM-99, Argonne National Laboratory (September 1987).

[15] Dongarra. J. \& R. van de (ieijn, Reduction to condensed form for the eigenvalue problem on distributed-memory architectures, Technical Report ORNL/TM-12006, Oak Ridge National Laboratory, Engineering Physics and Mathematics Division (January 1992).

[16] Fox, (i., M. Johnson, (i. Lyzenga, S. Otto, J. Salmon, \& D. Walker, Solving Problems on Concurrent Processors, Vol. I, Prentice-Hall, Englewood ('liffs. N.J. 1988.

[17] (iolub, (i. \&. ('. F. Van Loan, Matrix Computations, and $\mathrm{ed}$. Johns Hopkins University Press, Baltimore. 1989.

[18] (iropp, William [). \& Barry Smith, Chameleon parallel programming tools users manual, ANL93/23, Argonne National Laboratory (March 1993).

[19] Hendrikson. B. \& D. Womble, The torus-wrap mapping for dense matrix calculations on massively parallel computers, SAND92-0792, Sandia National Laboratories (1992).
[20] Howland, J. L., The sign matrix and the separation of matrix eigenvalues, Lin. Alg. Appl. 49 (1983), 221-32.

[21] Huo, Y. \& R. Schreiber, Efficient, massively parallel eigenvalue computation, RIAC'S Technical Report 93.02, Research Institute for Advanced Computer Science (1993).

[22] Huss-Lederman, S., E. M. Jacobson, A. Tsao, \& G. Zhang, Matrix Multiplication on the Intel Touchstone Delta, Technical Report SRC-TR93-101, Supercomputing Research ('enter (1993), (also PRISM Working Note \#11).

[23] Huss-Lederman, S., A. Tsao, \& G. Zhang, A Parallel Implementation of the Invariant Subspace Decomposition Algorithm for Dense Symmetric Matrices, Proceedings, Sixth SIAM Conference on Parallel Processing for Scientific Computing (Norfolk, Virginia, March 22-24, 1993) (R. F. Sincovec, eds.), SIAM, Philadelphia, 1993, (also PRISM Working Note \#9).

[24] Ipsen, I. \& E. Jessup, Solving the symmetric tridiagonal eigenvalue problem on the hypercube, Tech. Rep. RR-548, Yale University (1987).

[25] Lang, B., A parallel algorithm for reducing symmetric banded matrices to tridiagonal form, SIAM J. Sci. Stat. Comp. 14 (1993), no. 6.

[26] Lederman, S., A. Tsao, \& T. Turnbull, A parallelizable eigensolver for real diagonalizable matrices with real eigenvalues, Technical Report TR91-042, Supercomputing Research Center (1991).

[27] Li, T.-Y., H. Zhang, \& X.-H. Sun, Parallel homotopy aigorithm for symmetric tridiagonal eigenvalue problems, SIAM J. Sci. Stat. Comput. 12 (1991), no. 3, 469-87.

[28] Mathur, K. K. \& S. L. Johnsson, Multiplication of matrices of arbitrary shape on a data parallel computer (1992), Thinking Machines (orporation, preprint, also released as Technical Report TR-216.

[29] Schreiber, R., Solving eigenvalue and singular value problems on an undersized systolic array: SIAM J. Sci. Stat. ('omput. 7 (1986). no. 2. 441-451.

[30] (i. W. Stewart, Updating a rank-revealing ULV decomposition, SIAM J. Matrix Anal. Appl. 14 (1993), no. 2.

[31] Tsao, A. \& T. Turnbull, A comparison of algorithms for banded matrix multiplication. Technical Report SR(-TR-093-092, Supercomputing Research (enter (1993), (also PRIS.M Working Note \#6). 

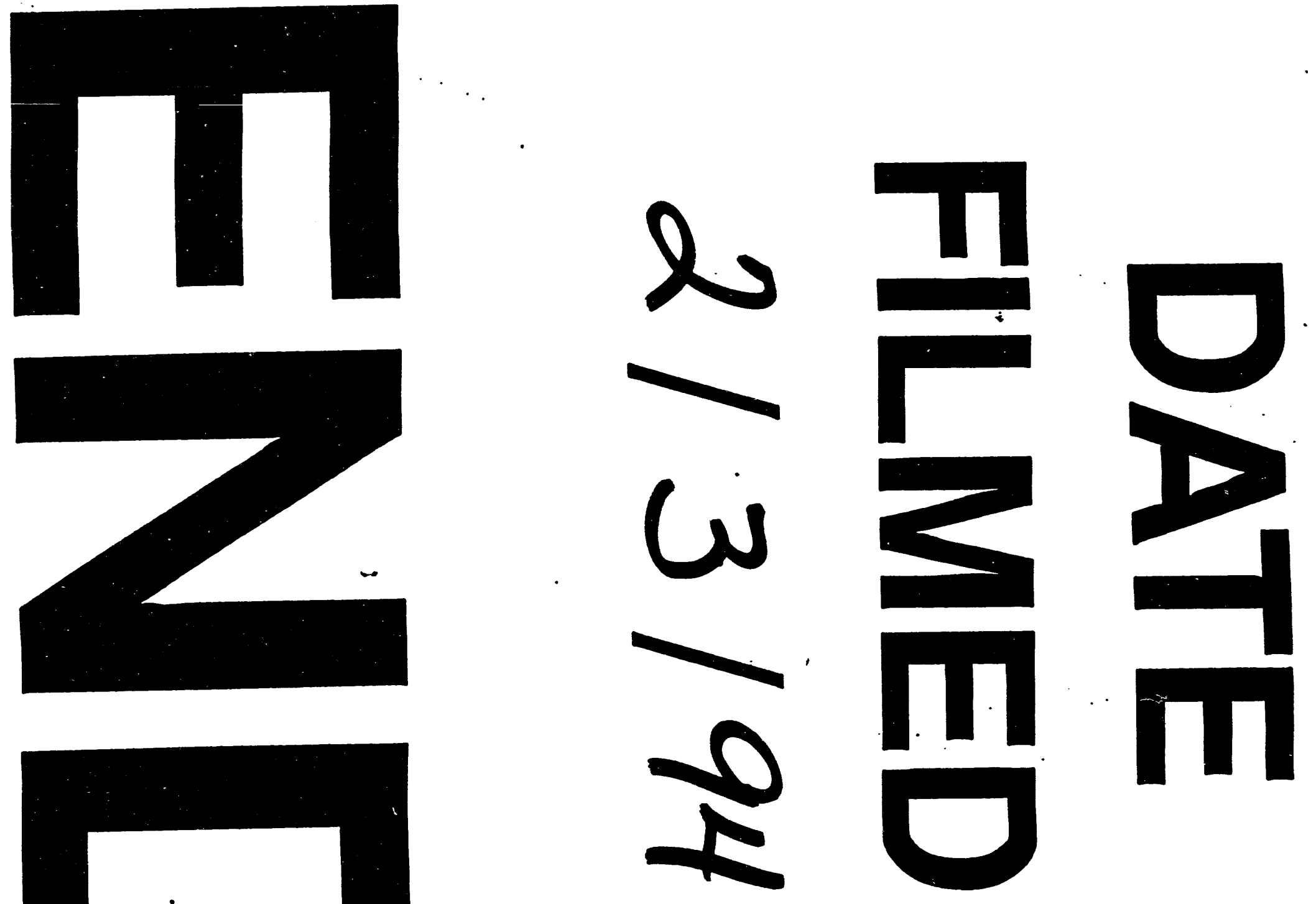